\title{
Dyslexia and hand preference in secondary school students
}

\author{
Filippos Vlachos, Eleni Andreou, Afroditi Delliou, and Paraskevi Agapitou \\ University of Thessaly, Volos, Thessaly, Greece
}

\begin{abstract}
Research results with regard to handedness and dyslexia have been ambiguous. The present study investigated the relationship between handedness and dyslexia in secondary school students based on genetic (Right-Shift) and hormonaldevelopmental theories of handedness. A total of 135 students (45 dyslexics and 70 age- and sex-matched controls) participated in the study. Handedness was defined according to the Edinburgh Handedness Inventory. We developed several classifications that represented various levels of handedness. Both continuous and dichotomous classifications of handedness revealed a small but reliable increase in the proportion of non-right-handers among dyslexics, likely because of the increased proportion of dyslexics among pure left-handers. Dyslexics did not display precisely the same pattern of right and left responses as controls, with some differences at the extremes of the continuum. The present results provide empirical support for Annett's (1985) Right-Shift theory predictions. Additionally, the present study indicates that using a numerical scoring system or dichotomous classifications with restricted criteria that permit the measurement of several degrees of handedness appears to better determine hand preference than using broad classifications into handedness groups. Keywords: dyslexia, hand preference, secondary school students.
\end{abstract}

Received 10 January 2013; received in revised form 17 January 2013; accepted 17 January 2013. Available online 27 June 2013.

\section{Introduction}

Dyslexia is the most common and carefully studied learning disability in school-age children (Shaywitz, Fletcher, \& Shaywitz, 1995). It is characterized by marked impairment in the development of reading skills and affects a large number of persons (Shastry, 2007).

Orton (1928) attempted to explain the genesis of reading disabilities by proposing a putative link between hand preference and developmental problems. The belief was that these deficits were somehow associated with atypical cerebral organization reflected by nonnormal patterns of handedness. The link between lefthandedness and dyslexia was boldly stated by Geschwind \& Behan (1982) who found an elevated incidence of selfreported dyslexia and stuttering, tabulated separately, in a large sample of strong left-handers. They reported that very strong left-handers were 11 times more likely to have dyslexia than very strong right-handers. Such findings led Geschwind \& Galaburda (1987) to propose their hormonal-developmental theory, postulating that a fundamental link exists between abnormal development of the left hemisphere, reduced dextrality, various

Filippos Vlachos, Afroditi Delliou, and Paraskevi Agapitou, University of Thessaly, Department of Special Education, Argonafton \& Filellinon, Volos, 38221, Greece. Eleni Andreou, University of Thessaly, Department of Primary Education, Argonafton \& Filellinon, Volos, 38221, Greece. Correspondence regarding this article should be directed to: Filippos Vlachos, University of Thessaly, Department of Special Education, Argonafton \& Filellinon, Volos, 38221, Greece. Phone: +30241074739. Fax:+30241074825. E-mail: fvlachos@uth.gr learning disorders, immune disorders, and other medical conditions, forged by the prenatal influence of fetal testosterone on the developing nervous system.

Other studies of dyslexics found the predicted excess of non-right-handers and that some dyslexics were strongly biased toward the right for hand skill (Annett \& Kilshaw, 1984). Annett \& Manning (1990) found an excess of poor readers at both the left and right of the hand skill continuum in a general school sample. These findings were consistent with the RightShift theory of Annett (1985), which suggested that the increase in mixed and left handers among dyslexics is attributable to the fact that some people with specific language problems lack the $r s+$ gene, which gives most people a left hemisphere advantage for speech and shifts the handedness distribution toward dextrality. According to Annett (1985), poor readers with poor phonology should be unbiased toward either side for hand skill. There should be more left handers and also more mixed handers as expected for a random distribution of handedness, without any shift to the right. The RightShift theory also predicts that there should be some poor readers who do not have problems with phonological processing but have other difficulties, probably with visual memory functions. The prediction was that the latter would be strongly biased toward dextrality as expected for the $r s+$ genotype.

Bishop (1990) reviewed 25 studies on handedness with regard to developmental disorders such as dyslexia and specific language impairment that met stringent methodological criteria and concluded that such an 
association had little support. Bishop (1990) stated that the results of Geschwind \& Behan (1982) were "totally discrepant with those reported by other workers" (Bishop, 1990, p. 147). She concluded that theories that predict differences in rates of left-handedness or the relative skill of two hands in either dyslexia or specific language impairment have little support. A reanalysis of the same studies using newer meta-analytical procedures (Eglinton \& Annett, 1994) showed a small but reliable increase in the proportion of non-right-handers among dyslexics as expected by the Right-Shift theory of handedness of Annett (1985).

A study by Tonnessen, Lokken, Hoien, \& Lundberg (1993) found relatively weak but supportive evidence that indicated an elevated prevalence of left-handedness in individuals with dyslexia. Another study by Locke \& Macaruso (1999) assessed manual laterality in dyslexic students and concluded that although the dyslexics did not show precisely the same pattern of right and left responses as controls, the differences between groups were few and not present at the extremes of the continuum (i.e., neither a significantly lower percentage of pure right-handers nor a higher percentage of pure left-handers was found among the dyslexics).

Based on the aforementioned studies, we can conclude that the research results on handedness and dyslexia are ambiguous at best, indicating a need for further clarification. Koufaki \& Papadatou-Pastou (2013) suggested that investigations of the relationship between handedness and reading disability must contend with a lack of consensus over the definition and measurement of handedness and the variability in the definition of dyslexia. According to Brenneman, Decker, Meyers, \& Johnson (2008), one of the reasons research on handedness and dyslexia has been ambiguous is that handedness is often measured as a dichotomous variable rather than as a continuous variable, and this methodological difference contributes to the diverse research findings. However, as indicated by Dragovic (2004), the majority of existing handedness questionnaires lack valid criteria for partitioning the continuous measure into a small number of handedness classes. That is the reason why researchers (e.g., Boscarino \& Hoffman, 2007; Giotakos, 2001; Taylor, Dalton, Fleminger, \& Lishman, 1982) sometimes use more than one criterion to test the stability of their findings or allow comparability with similar studies. To overcome this methodological problem, the present study evaluated the association between lateral preference for handedness and developmental dyslexia using both continuous and several dichotomous classifications of hand preference.

The hypotheses explored in the present study were based on the two aforementioned theories of handedness. More specifically, if the delayed development of the left hemisphere in left-handers, as proposed by Geschwind \& Galaburda (1987), is correct, then there should be a significant preponderance of left-handed students among dyslexics (Hypothesis 1). Conversely, according to the expectation of the Right-Shift theory of Annett (1985), we could predict an increase in mixed and lefthanders (i.e., non-right-handers) among dyslexics and an increased percentage of strong dextrality in dyslexics compared with controls (Hypothesis 2).

\section{Methods \\ Participants}

A total of 135 secondary school students (102 boys and 33 girls; age range 13-18 years) participated in the study. The dyslexic students $(n=45$; age range $13-18$ years; $M=15.14$ years, $S D=1.46$ years) had a statement of dyslexia after assessment at the Centre of Diagnosis, Assessment and Support of Magnesia, Greece. This center belongs to the Ministry of Education and is listed among the formal assessment centers for specific learning difficulties. The assessment was performed by a psychologist and special educator using the following criteria: (a) assessment of intelligence, (b) assessment of cognitive skills (i.e., visual discrimination, visual and auditory short-term memory, spatial orientation, laterality, etc.), and (c) assessment of oral reading accuracy, reading rate, reading comprehension, listening comprehension, dictation, and free writing using informal reading inventories. Students with dyslexia had a consistent history of persistent specific literacy difficulties, with reading levels at least 18 months behind their chronological age but with an Intelligent Quotient $>80$ on the Greek version of the Wechsler Intelligence Scale for Children-Revised ( $3^{\text {rd }}$ edition; WISCIII-R). A comparison group $(n=90$; age range $13-18$ years; $M=15.05$ years, $S D=1.49$ years) consisted of pupils who attended the same classes as the dyslexics and did not have a history of major medical illness, psychiatric illness, developmental disorder, or significant visual or auditory impairments according to the medical reports of their schools. Participants in the control group were matched for age and gender with the dyslexics. All of the participants had Greek as their first language and attended mainstream public schools.

\section{Measures}

Handedness was defined according to the Edinburgh Handedness Inventory (EHI; Oldfield, 1971), a reliable and well-validated instrument(Bryden, 1977). Numerous studies have confirmed the objectivity and reliability of the EHI with other handedness questionnaires (e.g., Dorthe, Blumenthal, Jason, \& Lantz, 1995; McMeekan \& Lishman, 1975; Ransil \& Schachter, 1994; Williams, 1986). The test-retest reliability of the EHI measured by the Pearson $r$, Kendall $\tau$, and Spearman $r_{s}$ ranged from 0.95 to 0.98 (Ransil \& Schachter, 1994). The medium to high correlations of the EHI with the other behavioral measures of handedness (e.g., the Purdue Pegboard test, a test of manual dexterity) underscore the high concurrent validity of the test (Raczkowski, Kalat, \& Nebes, 1974; Triggs, Calvanio, Levine, Heaton, \& Heilman, 2000; Verdino \& Dingman, 1998). Additionally, the Citation 
Index indicates that the EHI has been the most widely used inventory in the literature. The questionnaire is composed of 10 items that pertain to hand preference in writing, drawing, throwing a ball, using scissors, a toothbrush, a knife (without fork), a spoon, and a broom (upper hand), striking a match, and opening a box.

\section{Procedure}

All participants were examined individually on the 10 items of the EHI (Oldfield, 1971). To avoid possible misunderstandings caused by reading deficiencies in the dyslexics, the examiner told the subject that some familiar activities were going to be named, and the subject was to demonstrate how he/she ordinarily performed them. Each item was preceded by the phrase, "Show me how you...," and the examiner wrote down the answer on the questionnaire. Responses were scored "right" or "left" based on the hand used to demonstrate the activity.

Based on these responses, we calculated the Laterality Quotient $(\mathrm{LQ}=[$ Right - Left $] /[$ Right + Left] $\times 100]$ ) for each participant, resulting in a score that ranged from -100 to +100 in which -100 indicated pure left-handedness and +100 indicated pure righthandedness.

We developed several classifications that represented various levels of handedness. In the first classification, using Oldfield's (1971) categorization, the students were divided as right-handers (EHI score +1 to +100 ) and left-handers (EHI score -100 to -1). A score of 0 indicated pure mix handedness. Given that Dragovic (2004) concluded that handedness is not a bimodal phenomenon because mixed-handedness exists as an autonomous handedness pattern entrenched along the continuum and between the two extremes of human handedness (i.e., left and right), we developed two more classifications using more restrictive criteria, aiming to create more detailed categorizations of our participants in the handedness groups. More specifically, we classified the respondents into three groups. Students who scored +50 to +100 were considered right-handers (EHI score +50 to +100 ). Students who scored -49 to +49 were considered mixed-handers. Students who scored -50 to -100 were considered left-handers. Finally, the students were divided into five additional groups as pure righthanders (EHI score +90 to +100 ), moderate right-handers (EHI score +50 to +89 ), mixed-handers (EHI score -49 to +49 ), moderate left-handers (EHI score -50 to -89 ), and pure left-handers (EHI score -90 to -100 ). Table 1 summarizes the aforementioned classifications.

\section{Results}

The mean EHI scores were $5.87(S D=6.81)$ and $7.10(S D=4.78)$ for the dyslexic and control groups, respectively. A $t$-test with EHI scores as the dependent variable and group (dyslexics vs. controls) as the independent variable was used. The analysis revealed that the difference in mean EHI scores between the dyslexic and control groups was not statistically significant $\left(t_{133}=-1.22, p>.05\right)$, indicating that the two groups did not present significant differences in their handedness scores.

Figure 1 shows the distribution of scores on the EHI for the dyslexic and control groups. Typical with handedness preference inventories, the distribution was J- shaped, with the majority of participants being completely right-handed in both groups. Because the data were non-normally distributed, Mann-Whitney test was used to compare the distribution of handedness scores for the two groups of participants. No significant difference was found $\left(\chi^{2}=21.70, d f=1, p>.05\right)$, indicating that the differences in the percentages of EHI scores between dyslexics and controls were not significant.

The prevalence of handedness in ourstudy population based on the three classifications is presented in Table 2. When the students were divided into two groups as right-handers (EHI +1 to +100$)$ and left-handers (EHI -100 to -1$)$ using the broadest definition of handedness, a higher percentage of left-handers was found in the dyslexic group ( $17.8 \%$ vs. $8.9 \%$ in the control group). This difference did not reach statistical significance $\left(\chi^{2}=2.27, d f=1, p>.05\right)$. When the students were divided into three groups of right-handers (EHI +50 to +100 ), mixed-handers (EHI -49 to +49 ) and left-handers (EHI -100 to -50) using a more restrictive criterion, a higher prevalence of left-handers was also found in the dyslexic group $(17.8 \%$ vs. $5.6 \%$ in the control group), but this difference also did not reach statistical significance $\left(\chi^{2}=4.01, d f=2, p>.05\right)$. Finally, when the students were divided into five handedness groups (pure right-handers, moderate right-handers, mixedhanders, moderate left-handers, and pure left-handers) using the most restrictive criterion, the analysis revealed

Table 1. Three dichotomous handedness classifications of the participants according to their EHI score

\begin{tabular}{|c|c|c|c|c|c|}
\hline \multirow[b]{2}{*}{ Classification } & \multicolumn{5}{|c|}{ Handedness classification } \\
\hline & Right-handers & $\begin{array}{c}\text { Moderate } \\
\text { right-handers }\end{array}$ & Mixed-handers & $\begin{array}{l}\text { Moderate } \\
\text { left-handers }\end{array}$ & Left-handers \\
\hline $1 \mathrm{st}$ & +1 to +100 & & 0 & & -1 to -100 \\
\hline 2nd & +50 to +100 & & -49 to +49 & & -50 to -100 \\
\hline 3nd & +90 to +100 & +50 to +89 & -49 to +49 & -50 to -89 & -90 to -100 \\
\hline
\end{tabular}

EHI, Edinburgh Handedness Inventory. 


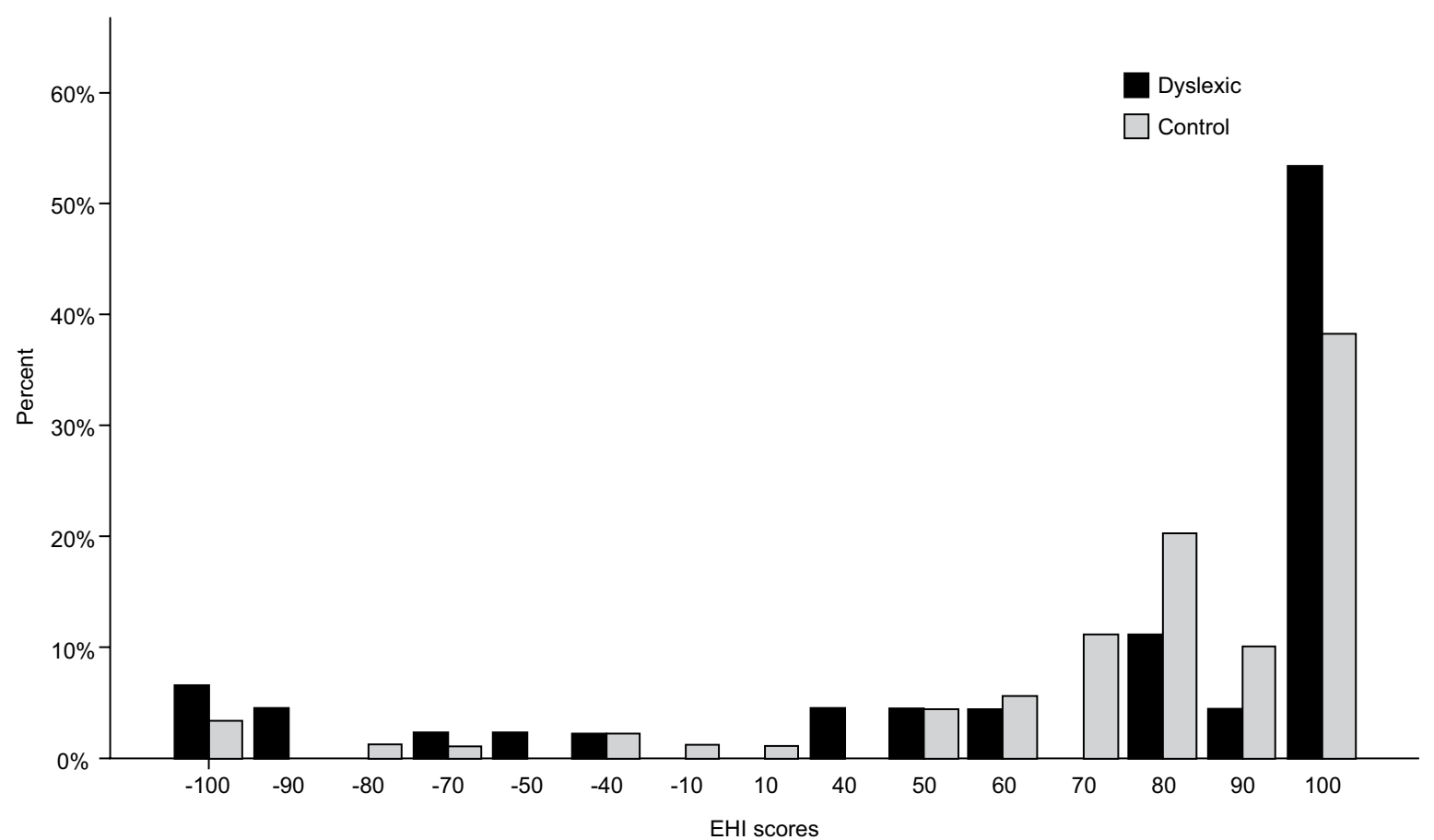

Figure 1. Percentages of Edinburgh Handedness Inventory (EHI) scores within the dyslexic and control groups.

Table 2. Percentage of students per handedness classification group within the control and dyslexic groups

\begin{tabular}{|c|c|c|c|c|c|}
\hline \multirow{2}{*}{ Group } & \multicolumn{5}{|c|}{ Handedness classification } \\
\hline & Right-handers & $\begin{array}{c}\text { Moderate } \\
\text { right-handers }\end{array}$ & Mixed-handers & $\begin{array}{l}\text { Moderate left- } \\
\text { handers }\end{array}$ & Left-handers \\
\hline Control & 91.1 & & & & 8.9 \\
\hline Dyslexic & 82.2 & & & & 17.8 \\
\hline Control & 90.0 & & 4.4 & & 5.6 \\
\hline Dyslexic & 77.8 & & 4.4 & & 17.8 \\
\hline Control & 47.8 & 42.2 & 4.4 & 3.4 & 2.2 \\
\hline Dyslexic & 57.8 & 20.0 & 4.4 & 6.7 & 11.1 \\
\hline
\end{tabular}

that the differences between controls and dyslexics were statistically significant $\left(\chi^{2}=9.98, d f=4, p<.05\right)$. More specifically, pairwise post hoc comparisons revealed that dyslexics were overrepresented not only in pure left-handers ( $11.1 \%$ in dyslexics $v s .2 .2 \%$ in controls) but also in pure right-handers $(57.8 \%$ in dyslexics $v s$. $47.8 \%$ in controls), whereas they were underrepresented in moderate right-handers $(20.0 \%$ in dyslexics $v s .42 .2 \%$ in controls).

\section{Discussion}

Using both continuous and several dichotomous classifications of hand preference in the present study, we investigated the relationship between handedness and dyslexia in Greek secondary school students based on genetic (Right-Shift) and hormonal-developmental theories of handedness.
Our first hypothesis predicted an increased incidence of left-handers among dyslexics based on the hormonal-developmental theory (Geschwind \& Galaburda, 1987), which correlated abnormal lefthemisphere development with learning disorders and non-right-handedness. Indeed, the two more broad dichotomous classifications of handedness revealed an elevated proportion of left-handers among dyslexics. This difference was in the direction expected but not statistically significant; therefore, we have to reject our first hypothesis.

The second hypothesis of the present study arose from Annett's (1985) Right-Shift theory. According to this hypothesis, the prediction was an increase in mixed- and left-handers (i.e., non-right-handers) among dyslexics and an increased percentage of strong dextrality in dyslexics compared with controls. Both 
the second and third dichotomous classifications of handedness confirmed this prediction, indicating more non-right-handers (i.e., left-handers plus moderate left-handers plus mixed-handers) in dyslexics than in controls. A careful observation of Table 2 reveals that overrepresentation of non-right-handers in dyslexics is mainly attributable to the fact that significantly more dyslexics $(11.1 \%)$ were pure left-handers compared with their matched controls (2.2\%). The continuous measure of handedness verified this finding. Figure 1 shows that the percentage of dyslexic students who scored -100 (pure left-handers) was higher than controls. Interestingly, the proportion of dyslexic students who scored +100 (pure right-handers) was also higher than controls. This finding was not detectable from the two more broad dichotomous classifications of handedness but emerged from both the third dichotomous and continuous classifications. The surprising idea that strong right-handers could be at risk for reading difficulties was first suggested by findings in a dyslexia clinical sample (Annett \& Kilshaw, 1984). The reliability of this finding was checked in a school sample when mean reading quotients were found to be lower at both ends of the laterality distribution (Annett \& Manning, 1990). The present results offer empirical support for Annett's (1985) Right-Shift theory predictions, confirming our second hypothesis.

Our results are also consistent with the results of the meta-analysis performed by Eglington \& Annett (1994), which found more sinistrals and mixedhanders throughout the dyslexia population, suggesting a probable relationship. A very recent meta-analysis (Koufaki \& Papadatou-Pastou, 2013) attempted to replicate and update the results of Eglinton \& Annett (1994). This updated meta-analysis included 44 studies and confirmed the statistically significant increase in nonright-handedness among dyslexics compared with nondyslexics, providing support for the hypothesis of specific language-associated brain function that is not adequately lateralized to the left hemisphere in people with dyslexia.

Our findings are consistent with previous studies (e.g., Tonnessen et al., 1993) that found a relationship between hand preference and dyslexia but not necessarily between left-handedness and dyslexia. However, our results do not support other studies (Peters, Reimers, \& Manning, 2006), which found that individuals who indicated "either" hand for writing preference had a significantly higher prevalence of dyslexia than individuals who had clear left or right hand preferences. A possible reason for this discrepancy is the way handedness was assessed. In the previous study, although the researchers conducted an internet study with a very large sample (i.e., $>250,000$ participants), they did not use a questionnaire to assess handedness. The participants answered only a graded question about which hand they preferred for writing, but this criterion is not sufficient to distinguish clear hand preference groups. Peters et al. (2006) concluded that contradictions in the literature about whether dyslexia or other behavioral variables are linked to handedness stem largely from different definitions of hand preference. According to Eglington \& Annett (1994), an additional reason for the inconclusive findings in the literature may be attributable to a failure to discriminate dyslexia subtypes. This assumption was confirmed by a recent review of findings on dyslexia and handedness (Annett, 2011), which concluded that phonological dyslexics are less likely to be right-handed, whereas surface or dyseidetic dyslexics are more likely to be right-handed compared with the general population.

The fact that we could not discriminate subtypes of dyslexia in our sample constitutes a limitation of our study. Another limitation is the small sample size that led to few mixed- and left-handers. Including more mixed- and left-handers would necessitate a much larger sample because the percentage of non-right-handers in the general population or in dyslexics is small.

Overall, our results indicate that dyslexics did not display precisely the same pattern of right and left responses as controls, with some differences at the extremes of the continuum. More specifically, both continuous and dichotomous classifications of handedness revealed a small but reliable increase in the proportion of non-right-handers among dyslexics, likely because of the increased proportion of dyslexics among pure lefthanders. Notwithstanding, there appears to be no reason to treat left-handed children as an at-risk population for dyslexia because an increased proportion of dyslexics was observed among pure right-handers as well.

Additionally, the present study indicates that using a numerical scoring system or dichotomous classifications with restricted criteria that permit the measurement of several degrees of handedness appears to better determine hand preference than using broad classifications into handedness groups.

In summary, the present results may allow us to draw a conclusion that is similar to Locke \& Macaruso (1999) who proposed that even if an elevated incidence of left-handedness or less-strong right-handedness exists in dyslexics, the typical dyslexic adolescent is strongly right-handed. This conclusion supports previous research findings (Natsopoulos, Kiosseoglou, Xeromeritou, \& Alevriadou, 1998) suggesting that left-handers consist of heterogeneous subgroups of subjects at either extreme of abilities, with significantly more subjects performing worse and significantly fewer subjects performing better than right-handers. The above argument appears to reinforce theoretical claims (Satz, Orsini, Saslow, \& Henry, 1985; Bishop, 1990) about the inclusion of a proportion of potentially pathological persons among nonclinical left-handers, even if they do not present overt neuromotor abnormalities. This may be the reason Gaillard \& Satz (1989) suggested that the association between handedness and ability depends on whether the cohort is selected from a general school population or from a clinical population. In the former case, null results are usually reported. In contrast, studies based on clinical samples have often reported a significant effect of handedness. 


\section{References}

Annett, M. (1985). Left, right, hand and brain: The right shift theory. Hillsdale, NJ: Erlbaum.

Annett, M. (2011). Dyslexia and handedness: Developmental phonological and surface dyslexias are associated with different biases for handedness. Perceptual \& Motor Skills, 112, 417-425.

Annett, M., \& Kilshaw, D. (1984). Lateral preference and skill in dyslexics: Implications of the right shift theory. Journal of Child Psychology and Psychiatry, 25, 357-377.

Annett, M., \& Manning, M. (1990). Reading and a balanced polymorphism for laterality and ability. Journal of Child Psychology and Psychiatry, 31, 511-529.

Bishop, D. V. M. (1990). Handedness and developmental disorders. Oxford, UK: MacKeith Press.

Boscarino, J. A., \& Hoffman, S. N. (2007). Consistent association between mixed lateral preference and PTSD: Confirmation among a national study of 2490 US Army Vietnam veterans. Psychosomatic Medicine, 69, 365-369.

Brenneman, M. H., Decker, S., Meyers, J., \& Johnson, K. (2008). Does a continuous measure of handedness predict reading processes and reading-related skills across the lifespan? Laterality, 13, 481-503.

Bryden, M. P. (1977). Measuring handedness with questionnaires. Neuropsychologia, 15, 617-624.

Dorthe, N. J., Blumenthal, T. D., Jason, D. R., \& Lantz, P. E. (1995). The use of next-of-kin in assessing handedness. Perceptual and Motor Skills, 81, 203-208.

Dragovic, M. (2004). Categorization and validation of handedness using latent class analysis. Acta Neuropsychiatrica, 2004, 212-218.

Eglinton, E., \& Annett, M. (1994). Handedness and dyslexia: A metaanalysis. Perceptual and Motor Skills, 79, 1611-1616.

Gaillard, F., \& Satz, P. (1989). Handedness and reading disability: A developmental study. Archives of Clinical Neuropsychology, 4, 63-69.

Geschwind, N., \& Behan, P. (1982). Left-handedness: Association with immune disease, migraine, and developmental learning disorder. Proceedings of the National Academy of Sciences of the United States of America, 79, 5097-5100.

Geschwind, N., \& Galaburda, A. M. (1987). Cerebral lateralization: Biological mechanisms, associations, and pathology. Cambridge, MA: MIT Press.

Giotakos, O. (2001). Narrow and broad definition of mixedhandedness in male psychiatric patients. Perceptual \& Motor Skills, 93, 631-638.

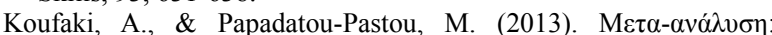

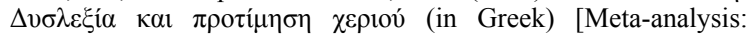
dyslexia and hand preference]. Proceedings of the 2nd Hellenic Conference of Educational Sciences, May 27-30, 2010. Athens: Smirniotakis.
Locke, J. L., \& Macaruso, P. (1999). Handedness in developmental dyslexia: Direct observation of a large sample. Journal of Neurolinguistics, 12, 147-156.

McMeekan, E. R., \& Lishman, W. A. (1975). Retest reliabilities and interrelationship of the Annett Hand Preference Questionnaire and the Edinburgh Handedness Inventory. British Journal of Psychology, 66, 53-59.

Natsopoulos, D., Kiosseoglou, G., Xeromeritou, A., \& Alevriadou, A. (1998). Do the hands talk on mind's behalf? Differences in language ability between left- and right-handed children. Brain and Language, 64, 182-214.

Oldfield, R. C. (1971). The assessment and analysis of handedness: The Edinburgh inventory. Neuropsychologia, 9, 97-113.

Orton, S. (1928). Specific reading disability: Strephosymbolia. Journal of the American Medical Association, 90, 1095-1099.

Peters, M., Reimers, R., \& Manning, J. T. (2006). Hand preference for writing and associations with selected demographic and behavioral variables in 255,100 subjects: The BBC internet study. Brain and Cognition, 62, 177-189.

Raczkowski, D., Kalat, J. W., \& Nebes, R. (1974). Reliability and validity of some handedness questionnaire items. Neuropsychologia, 12, 43-47.

Ransil, B. J., \& Schachter, S. C. (1994). Test-retest reliability of the Edinburgh Handedness Inventory and Global Handedness preference measurements, and their correlation. Perceptual and Motor Skills, 79, 1355-1372.

Satz, P., Orsini, D. L., Saslow, E., and Henry, R. (1985). The pathological left-handedness syndrome. Brain and Cognition, 4, 27-46.

Shastry, B. (2007). Developmental dyslexia: An update. Journal of Human Genetics, 52, 104-109.

Shaywitz, B. A., Fletcher, J. M., \& Shaywitz, S. E. (1995). Defining and classifying learning disabilities and attention-deficit/hyperactivity disorder. Journal of Child Neurology, 10(Suppl. 1), S50-S57.

Taylor, P. J., Dalton, R., Fleminger, J. J., \& Lishman, W. A. (1982). Differences between two studies of hand preference in psychiatric patients. British Journal of Psychiatry, 140, 166-173.

Tonnessen, F. E., Løkken, A., Høien, T., \& Lundberg, I. (1993). Dyslexia, left-handedness, and immune disorders. Archives of Neurology, 50, 411-416.

Triggs, W. J., Calvanio, R., Levine, M., Heaton, R. K., \& Heilman, K. M. (2000). Predicting hand preference with performance on motor tasks. Cortex, 36, 679-689.

Verdino, M., \& Dingman, S. (1998). Two measures of laterality in handedness: The Edinburgh Handedness Inventory and the Purdue Pegboard test of manual dexterity. Perceptual and Motor Skills, $86,476-478$

Williams, S. (1986). Factor analysis of the Edinburgh Handedness Inventory. Cortex, 22, 325-326. 